\title{
Life-threatening Capillary Leak Syndrome in an Adult with Refractory Acute Myeloid Leukemia during Allogeneic Transplantation: a Case Report and Review of Literature
}

\author{
Yi-Zhi J, Lai-Quan H, Gui-Ping S, Yan D, He-Sheng $H$ and Dong-Ping $H^{*}$ \\ Department of Hematology, The Affiliated Yijishan Hospital of Wannan Medical College, Wuhu, China
}

\begin{abstract}
Background: Although allogeneic hematopoietic stem cell transplantation (allo-HSCT) offers the possibility of cure for hematological malignancies, various complications have been described. Capillary leak syndrome (CLS) has been previously observed in HSCT patients. CLS is a rare disease characterized by recurrent episodes of generalized edema and severe hypotension along with hypoproteinemia. Case Report: A 27-year-old Chinese man, diagnosed with refractory acute myeloid leukemia, was treated with a haploidentical stem cell transplant combined with an unrelated umbilical cord blood unit. The patient developed fatal CLS during the 9th day of the conditioning therapy.

Conclusion: Since it is difficult to distinguish between CLS and other early complications during allo-HSCT, our report highlights the need for rigorous investigation of identifying CLS and the increasing need of insightful diagnosis to manage any incidence of CLS.
\end{abstract}

Keywords: Acute myeloid leukemia; Allogeneic transplantation; Capillary leak syndrome; Endothelial damage; Early complications

\section{Introduction}

CLS is one of the life-threatening early complications which usually occur during hematopoietic stem cell infusion or hematopoietic reconstruction process in addition to graft-versus-host-disease (GVHD) and infection [1]. It is characterized by unexplained episodic capillary hyperpermeability, which causes the shift of fluid and protein from the intravascular space to the interstitial space [2]. However, since the nonspecific signs and symptoms of CLS and the overlapping manifestations of early complications after transplantation, CLS tends to be easily confused with other early complications for clinicians. In this case, we report an adult with refractory acute myeloid leukemia who developed fatal CLS during allo-HSCT with review of the literature.

\section{Case Report}

A 27-year-old male was first admitted to our hospital in August 2014 with complaints of chills and fever. He exhibited obvious pain and swelling of gastrocnemius and activity abstacle. Peripheral blood counts revealed white cell counts of $29.9 \times 10^{\%} / \mathrm{L}$, hemoglobin lever of $89 \mathrm{~g} / \mathrm{L}$, platelet counts of $179 \times 10^{9} / \mathrm{L}$. Bone marrow was hypercellular exhibiting infiltration with $30 \%$ blast cells comprising myeloblasts and promonocytes. Immunophenotype analysis showed 54\% abnormal cells which were positive for CD13, HLA-DR, CD11b, CD11c, CD33, CD14, CD64 and CD15, and weakly positive for CD34 and MPO. The overall findings were consistent with acute myeloid leukemia. G-banding revealed 46, XY. Moreover, genetic testing revealed positive for dupMLL fusion. He did not respond to "HA" $(\mathrm{HHT} 4 \mathrm{mg} / \mathrm{d} \times 7 \mathrm{~d}$, Ara-c $0.2 \mathrm{~g} / \mathrm{d} \times 7 \mathrm{~d}$ ) and subsequent "IA" (IDA $30 \mathrm{mg} \mathrm{d} 1,20 \mathrm{mg} \mathrm{d} 2-3$, Ara-c $0.2 \mathrm{~g} / \mathrm{d} \times 7 \mathrm{~d}$ ) induction chemotherapy.

Salvage therapy consisted of DAC (decitabine) $\left(20 \mathrm{mg} / \mathrm{m}^{2} / \mathrm{d} \times 5 \mathrm{~d}\right)$, Ara-c (cytarabine) $\left(10 \mathrm{mg} / \mathrm{m}^{2} / \mathrm{d} \times 2 \mathrm{~d}\right)$ and Ara-c $\left(10 \mathrm{mg} / \mathrm{m}^{2}\right.$ every 12 $\mathrm{h} \times 3 \mathrm{~d}$ ) was planned. Because no full HLA-matched donor was readily available, a combination of a haploidentical stem cell graft and an unrelated umbilical cord blood unit was scheduled. The BU/CY-based conditioning regimen consisted of Me-CCNU $250 \mathrm{mg} / \mathrm{m}^{2}$ (day -10), Ara-c $4 \mathrm{~g} / \mathrm{m}^{2} /$ day (days -9 and -8 ), Bu $4 \mathrm{mg} / \mathrm{kg} /$ day (days -7 to -5 ),
CTX $1.8 \mathrm{~g} / \mathrm{m}^{2} /$ day (days -4 and -3 ) and $\mathrm{r}$-ATG (rabbit antithymocyte globulin) $2.5 \mathrm{mg} / \mathrm{kg}$ (days -5 to -2 ).

The number of infused nucleated cells and CD34+/CD45+ cells were $21.75 \times 10^{8} / \mathrm{kg}$ and $2.33 \times 10^{6} / \mathrm{kg}$ for haploidentical transplantation and $0.184 \times 10^{8} / \mathrm{kg}$ and $1.35 \times 10^{5} / \mathrm{kg}$ for cord blood transplantation.

On the ninth day of the conditioning therapy, he developed palpitation, breathlessness, oliguria and progressive edema of his face and four limbs. But his blood urea nitrogen (BUN $5.77 \mathrm{mmol} / \mathrm{L}$ ) and creatinine $(55.3 \mu \mathrm{mol} / \mathrm{L})$ were in normal ranges. The following day at 8:00 AM, he developed generalized edema and BUN and creatinine levels began to rise (BUN $15.38 \mathrm{mmol} / \mathrm{L}$ and creatinine $87.9 \mu \mathrm{mol} / \mathrm{L}$ ) accompanied with hypoalbuminemia (total protein/albumin 45.5/25.1 $\mathrm{g} / \mathrm{L}$ ). On physical examination, his temperature was $37.4^{\circ} \mathrm{C}$, blood pressure (BP) was low $(70 / 45 \mathrm{mmHg})$, central venous pressure was only $3 \mathrm{~cm} / \mathrm{H}_{2} \mathrm{O}$, heart beats were 140 beats/min and oxygen saturation decreased to $80 \%$. The electrocardiogram (ECG) showed sinus tachycardia.

He had no painful hepatomegaly and ascites suggesting venoocclusive disease. As these findings pointed out CLS, the patient was resuscitated with fluid infusion under intensive care and appropriate diuretic to relieve the edema. Prophylactic therapy with macromolecule hetastarch was done to improve colloid osmotic pressure. methylprednisolone was administered to improve the

*Corresponding author: Dong-Ping Huang, Department of Hematology, The Affiliated Yijishan Hospital of Wannan Medical College, West Zheshan Road 2, Wuhu 241001, China, Tel: 86-13955309713; E-mail: hdp0513@163.com

Received December 22, 2015; Accepted December 30, 2015; Published December 31, 2015

Citation: Yi-Zhi J, Lai-Quan H, Gui-Ping S, Yan D, He-Sheng H, et al. (2015) Lifethreatening Capillary Leak Syndrome in an Adult with Refractory Acute Myeloid Leukemia during Allogeneic Transplantation: a Case Report and Review of Literature. J Transplant Technol Res S4: 002. DOI: 10.4172/2161-0991.1000S4002

Copyright: ( 2015 Yi-Zhi J, et al. This is an open-access article distributed under the terms of the Creative Commons Attribution License, which permits unrestricted use, distribution, and reproduction in any medium, provided the original author and source are credited. 
Citation: Yi-Zhi J, Lai-Quan H, Gui-Ping S, Yan D, He-Sheng H, et al. (2015) Life-threatening Capillary Leak Syndrome in an Adult with Refractory Acute Myeloid Leukemia during Allogeneic Transplantation: a Case Report and Review of Literature. J Transplant Technol Res S4: 002. DOI: 10.4172/2161-0991.1000S4-002

capillary permeability, relieve the capillary leak, and to ensure the perfusion of major organs. CVP was closely monitored. Clinically, the patient progressively improved. However, he redeveloped severe edema and anuria on day 30 . BNP, liver enzymes and serum bilirubin lever continued to increase. Neutrophil engraftment was achieved on day 33. After sequential hemofiltration, he developed poor progress with deteriorating renal and liver function, resulting in death on day 42. A documented infectious cause was found in this episode. On the tenth day he developed a high fever but quickly controlled after empiric antibiotics (imipenem and vancomycin) were administered. His blood and catheter cultures were positive for Klebsiella pneumoniae.

\section{Discussion}

Allo-HSCT is a curative procedure for a subset of patients with hematologic malignancies. However, allo-HSCT is still associated with high treatment-related mortality due to severe infections and noninfectious complications. Especially during hematopoietic stem cell infusion or hematopoietic reconstruction process, a constellation of symptoms and signs including fever, erythrodermatous skin rash, and noncardiogenic pulmonary can be potentially fatal. CLS is a severe early complication of HSCT characterized by weight gain, generalized edema, hypotension, and hypoalbuminemia [2]. We present an alloHSCT AML patient affected by fatal CLS during conditioning therapy with a short survival in which the crises have disappeared temporarily after treatment but eventually died of CLS recurrence.

CLS can be secondary to HSCT [3] including autograft, allograft and non-myeloablative transplantation, severe infections, trauma, post extracorporeal circulation [4] especially infants after extracorporeal circulation and some cytokines [5,6] such as IL-2, G-CSF and GMCSF. The main CLS pathogenesis is injury of the capillary endothelium resulting in a loss of intravascular fluid into interstitial spaces [7]. Its mechanism is unknown, but in many studies vascular endothelial growth factor (VEGF) is the most suspected potent inducer of vascular permeability and may have a crucial role in the mechanism underlying CLS formation [8]. It is a serious condition and can be potentially fatal if not treated at the right time and with appropriate therapy. CLS after HSCT has been difficult to ameliorate. Treatment is limited to withdrawal of growth factors and systemic corticosteroids. The main aim of the treatment in the acute phase of CLS is to maintain tissue perfusion and to protect organs from the effect of the shock $[2,9]$. Yabe et al reported that bevacizumab may have a broad spectrum of efficacy against the life-threatening complication during HSCT [10]. In addition to the treatment for the acute phase, prevention of this is also important. Several prophylactic therapies such as Beta-2 stimulators (terbutaline or salbutamol) and theophylline have been tried, but their efficacy remains unclear [2].

The reported clinical presentation of CLS was varied primarily due to the lack of uniform diagnostic criteria. Currently, the diagnosis of CLS is still rely on clinical information, generally according to the following points 1) the cause of CLS, such as severe infection, chemotherapy drugs, CsA and cytokines such as interleukin-2, G-CSF and GM-CSF, 2) progressive systemic edema, weight gain, oliguria even anuria, dyspnea, drop of BP and CVP, severe edema after albumin supplementation and poor therapeutic efficacy after diuretic, 3) laboratory tests reveal that hypoalbuminemia, hypoxemia, creatinine and BUN increase progressively [11].

In our case, the patient was clinically diagnosed as CLS because of unexplained hypotension, diffuse edema, severe hypoalbuminemia, progressive oliguria and weight gain that occurred suddenly during the conditioning therapy. Infections and high-dose cytotoxic drugs rather than allograft were considered to be the common event due to the symptoms first appeared before allograft implanted. We treated our patient with intravenous fluid therapy $\left(4 \mathrm{~L} / \mathrm{m}^{2}\right)$ and albumin $(1 \mathrm{~g} / \mathrm{kg})$. In addition to the fluid therapy, we also treated him with methylprednisolone $(2 \mathrm{mg} / \mathrm{kg} /$ doses $)$ and immunoglobulin $(1 \mathrm{~g} / \mathrm{kg})$. His symptoms disappeared temporarily while he redeveloped severe edema and anuria on day 30 . We speculated that the recurrence of CLS might be associated with the following factors. Firstly, he was a patient with refractory acute myeloid leukemia and did not achieve complete remission (CR) before allo-HSCT. Secondly, he received high-dose chemotherapy consisted of various cytotoxic drugs. Last but not least, he developed blood poisoning after CLS. All the disadvantages might accelerate the development of CLS. He finally died of multiple organ failure despite early recognition of the syndrome and prompt resuscitation.

Although acute GVHD is a major complication appear soon after allo-HSCT, patients often experience other serious non-infectious complications, such as hepatic veno-occlusive disease (VOD), transplant-associated microangiopathy (TAM), intestinal TAM (iTAM), engraftment syndrome (ES), hemophagocytic syndrome, idiopathic pneumonia syndrome (IPS), diffuse alveolar hemorrhage (DAH), and CLS. All these non-infectious complications share the following characteristics: they have an early onset after HSCT, overlapping clinical manifestations, the absence of well-defined clinical criteria for diagnosis (and consequently an unknown true incidence), the absence of well-established treatments, and the tendency to evolve to an irreversible multiorgan dysfunction syndrome [12]. The similar presentation and management of CLS to ES, VOD or GVHD has therefore made their distinction difficult in allogeneic setting [13]. In our case, the symptoms first appeared was prior to engratment. In addition, the findings related to skin rash, pruritis, dyspnea, angioedema, painful hepatomegaly and liver damage were not present as the initial symptoms. Hence, we excluded ES, VOD and GVHD decisively.

Recent studies indicated that endothelial injury seems to be the initiating event in the cascade of events leading to their overlapping clinical manifestations of the early complications [14]. During HSCT, endothelial cells (ECs) are activated and damaged by several factors, including conditioning, cytokines released by damaged tissues, endotoxins translocated through damaged mucosa [15], drugs used in the procedure (such as G-CSF or calcineurin inhibitors) [16,17], the engraftment, and in the allogeneic setting-immunological reactions [18]. The different clinical syndromes that

occur could be determined by the predominant phenotypic change in the ECs and the location of this change (organ dependant or systemic) [19]. Several translational studies have provided evidence of this endothelial dysfunction on the basis of analysis of soluble markers, soluble forms of adhesion molecules, the enumeration of circulating ECs and microparticles, and morphologic and functional changes induced in cultured ECs [20]. Besides, Norihiro et al, [21] reported that the high ANG2 level at transplant was significantly associated with the increased incidence of the non-infectious complications and poor survival.

In conclusion, this report demonstrates fatal CLS can occur at each phase especially during hematopoietic stem cell infusion or hematopoietic reconstruction process. When sudden systematic edema and severe hypotension not reacting to hyperensort occur, evaluation and treatment are required to be performed with consideration of the 
Citation: Yi-Zhi J, Lai-Quan H, Gui-Ping S, Yan D, He-Sheng H, et al. (2015) Life-threatening Capillary Leak Syndrome in an Adult with Refractory Acute Myeloid Leukemia during Allogeneic Transplantation: a Case Report and Review of Literature. J Transplant Technol Res S4: 002. DOI: 10.4172/2161-0991.1000S4-002

possibility of CLS. To identify CLS from other early complications during HSCT and find out optimal management of it should be future goals.

\section{Patient Consent}

Written informed consent was obtained from the patient's parents for publication of this case report.

\section{References}

1. Cahill RA, Spitzer TR, Mazumder A (1996) Marrow engraftment and clinica manifestations of capillary leak syndrome. Bone marrow Transplant 18: 177-184.

2. Kawabe S, Saeki T, Yamazaki H, Nagai M, Aoyagi R, et al. (2002) Systemic capillary leak syndrome. Intern Med 41: 211-215

3. Nurnberger W, Willers R, Burdach S, Gobel U (1997) Risk factors for capillary leak syndrome after bone marrow transplantation. Ann Hematol 74: 221-224.

4. Abrahamov D, Erez E, Tamariz M, Dagan O, Pearl E, et al. (2002) Plasma vascular endothelial growth factor level is a predictor of the severity of postoperative capillary leak syndrome in neonates undergoing cardiopulmonary bypass. Pediatri Surg Int 18: 54-59.

5. Oeda E, Shinohara K, Kamei S, Nomiyama J, Inoue H (1994) Capillary leak syndrome likely the result of granulocyte colony-stimulating factor after highdose chemotherapy. Intern Med 33: 115-119.

6. Shinohara K (2011) Systemic capillary leak syndrome caused by granulocyte colony-stimulating factor. Intern Med 50: 2259.

7. Zancanaro A, Serafini F, Fantin G, Murer B, Cicardi M, et al. (2015) Clinical and pathological findings of a fatal systemic capillary leak syndrome (Clarkson disease) a case report. Medicine (Baltimore) 94: e591.

8. Grove CS, Lee YC (2002) Vascular endothelial growth factor: the key mediator in pleural effusion formation. Curr Opin Pulm Med 8: 294-301

9. Dhir V, Arya V, Malav IC, Suryanarayanan BS, Gupta R, et al. (2007) Idiopathic systemic capillary leak syndrome (SCLS) case report and systematic review of cases reported in the last 16 years. Intern Med 46: 899-904.
10. Yabe H, Yabe M, Koike T, Shimizu T, Morimoto T, et al. (2010) Rapid improvement of life-threatening capillary leak syndrome after stem cell transplantation by bevacizumab. Blood 115: 2723-2724.

11. Marx G, Vangerow B, Burczyk C, Gratz KF, Maassen N (2000) Evaluation of noninvasive determinants for capillary leakage syndrome in septic shock patients. Intensive Care Med 26: 1252-1258.

12. Carreras E, Apperley J, Carreras E, Gluckman E, Gratwohl A, et al. (2008) Early complications after HSCT Haematopoietic stem cell transplantation. Forum Service Editore: Genova. 180-195.

13. Spitzer TR (2001) Engraftment syndrome following hematopoietic stem cell transplantation. Bone Marrow Transplant 27: 893-898.

14. Carreras E, Diaz-Ricart M (2011) The role of the endothelium in the short term complications of hematopoietic SCT. Bone Marrow Transplant 46: 1495-1502.

15. Eissner G, Multhoff G, Holler E (1998) Influence of bacterial endotoxin on the allogenicity of human endothelial cells. Bone Marrow Transplant 21: 12861288.

16. Fuste B, Mazzara R, Escolar G, Merino A, Ordinas A, et al. (2004) Granulocyte colony-stimulating factor increases expression of adhesion receptors on endothelial cells through activation of p38 MAPK. Haematologica 89: 578-585.

17. Mercanoglu F, Turkmen A, Kocaman O, Pinarbasi B, Dursun M (2004) Endothelial dysfunction in renal transplant patients is closely related to serum cyclosporine levels. Transplant Proc 36: 1357-1360.

18. Cooke KR, Jannin A, Ho V (2008) The contribution of endothelial activation and injury to end-organ toxicity following allogeneic hematopoietic stem cell transplantation. Biol Blood Marrow Transplant 14: 23-32.

19. Aird WC (2008) Endothelium in health and disease. Pharmacol Rep 60: 139-143.

20. Cutler C, Kim HT, Ayanian S, Bradwin G, Revta C et al. (2010) Prediction of veno-occlusive disease using biomarkers of endothelial injury. Biol Blood Marrow Transplant 16: 1180-1185

21. Ueda N, Chihara D, Kohno A, Tatekawa S, Ozeki K, et al. (2014) Predictive value of circulating angiopoietin-2 for endothelial damage-related complications in allogeneic hematopoietic stem cell transplantation. Biol Blood Marrow Transplant 20: 1335-1340. 